\title{
MODELLING OF PULSATING DIFFUSIONAL BOUNDARY LAYERS-I.* A REVERSIBLE METAL-METAL ION ELECTROCHEMICAL REACTION UNDER SYMMETRIC SQUARE WAVE PERTURBING POTENTIALS AT A PLANE ELECTRODE
}

\author{
S. L. Marchiano, ${ }^{\dagger}$ L. Rebollo Neira ${ }^{\ddagger}$ and A. J. ARVia ${ }^{\dagger}$
}

\begin{abstract}
${ }^{\dagger}$ Instituto de Investigaciones Fisicoquímicas Teóricas y Aplicadas (INIFTA), Facultad de Ciencias Exactas, Universidad Nacional de La Plata, Sucursal 4, Casilla de Correo 16, $1900 \mathrm{La}$ Plata, Argentina ‡Departamento de Física, Universidad Nacional de La Plata, Casilla de Correo 67 (1900) La Plata, Argentina
\end{abstract}

(Received 13 December 1988; in revised form 10 May 1988)

\begin{abstract}
The concentration profiles of reacting species at the electrode-solution interface under a periodic square wave perturbing potential are calculated by solving the diffusion equation for the case of a fast reversible metal-metal ion reaction. This situation is particularly interesting in the electrochemical facetting of metals. The model explains the dependence of current density and charge density on the frequency and number of cycles of the perturbing potential.
\end{abstract}

\section{INTRODUCTION}

Periodic perturbations, either current or potential have been used in electrochemistry in connection to electrochemical techniques such as ac voltammetry, and impedance measurements $[1,2]$ as well as electrolysis with a pulsating current [3-9]. The latter has been extensively used in pulse plating, electrodissolution (electrochemical shaping and polishing of metals) anodizing and $a c$ corrosion processes. Pulsed current can produce considerable modifications on electrodeposits as far as the morphology, uniformity, compactness and adherence to the substrate are concerned. These effects are largely due to the influence of the periodic perturbation on the mass transport processes and accordingly, on the kinetics of the electrode reaction. The periodic perturbation produces a pulsating diffusional boundary layer wherein the concentrations of the ionic species oscillate with time near the electrode surface. This means that for instance, for a pulsating current the concentration and surface reaction overpotential depend not only on the magnitude but also on the frequency of the oscillating current.

Recently the application of periodic perturbing potentials under certain well-established conditions to various noble metal electrode has allowed study of the electrochemical facetting of those electrode surfaces [10-12]. Electrochemical facetting requires an anodic and a cathodic potential limit located at the negative and positive potential values, respectively, of the equilibrium potential of the corresponding metal-metal ion redox couple, to allow the electrodissolution and electrodeposition of the metal during the potential cycling. It has been demonstrated that the two processes are not exactly symmetric from the standpoint of the charge playing part in the oxidation-reduction cycles and this leads to an accumulation of dissolved metal in solution in the course of

*Part II: Electrochimica Acta, Vol. 35, No. 1, pp. 215-223 (1989). the electrochemical treatment. This has been clearly proved by chemical analysis, rotating disc and ring-disc electrode techniques for various metals [13]. Therefore, the electrochemical facetting implies the build-up of concentration profiles related to the anodic and cathodic reactions, that is the existence of a pulsating diffusional boundary layer.

For process modelling let us firstly assume that the overall electrochemical reaction involves a redox process under ionic diffusion control in the solution phase. Hence, to solve this problem the analytical solution of the Fick's diffusion equation is required to obtain the concentration profile, current density and charge density at any cycle. This can be done exclusively on the basis of the pure diffusion equations because as the metal ion concentration initially is null and along the process is much smaller than the acid electrolyte concentration, migration effect can be neglected. Analogously, provided that the period of the perturbing potential is adequately chosen and the electrode size is sufficiently small, it is possible to suppose that convection and double layer effects can be also disregarded[14]. It is further assumed that the values of $j_{o}$, the exchange current density for the metal-metal ion electrochemical reaction, is sufficiently large to avoid any activation polarization.

Furthermore, the perturbing potential is a periodic symmetric square wave potential for which the boundary conditions of the problem can be easily established.

The purpose of this work is to find out the dependence of the concentration profiles on frequency and number of cycles of the periodic potential and simplest electrochemical kinetic conditions.

\section{MATHEMATICAL MODELLING}

Let us consider that the following reaction takes place at a planar solid electrode (M) immersed in an 
acid electrolyte:

$$
\mathbf{M}=\mathbf{M}^{z+}+z \mathrm{e}^{-} .
$$

Reaction (1) is assumed to be under diffusional control as the corresponding exchange current density value $j_{0}$, is large. Therefore, for a semi-infinite diffusion at a plane electrode the concentration profile of the reacting species, can be obtained by solving Fick's equation:

$$
\frac{\partial c_{i}}{\partial t}=D_{i} \frac{\partial^{2} c_{i}}{\partial x^{2}}
$$

where $c_{i}$ corresponds to the concentration of the $\mathrm{M}^{2+}$ species, its diffusion coefficient being $D_{i}$. The diffusion of $\mathrm{M}^{z+}$ takes place only along the $x$-axis, ie the direction perpendicular to the electrode surface. The electrode is subjected to a symmetric square wave perturbing potential at frequency $f=1 / T=1 / 2 \tau$, where $T$ and $\tau$ being the period and half period, respectively. The upper, $E_{\mathrm{a}}$, and the lower, $E_{\mathrm{c}}$, potential limits are selected as, $E_{\mathrm{a}}>E_{\mathrm{r}}$ and $E_{\mathrm{c}}<E_{\mathrm{r}}, E_{\mathrm{r}}$ being the equilibrium potential of reaction (1). This perturbing potential brings about time-dependent boundary conditions to equation (2). Thus, the initial and boundary conditions are:

$t=0 \quad c_{i}=0$

$t>0 \quad\left\{\begin{array}{ll}x=0 & c_{i}=f(t) \\ x \rightarrow \infty & c_{i}=0\end{array}\right.$,

where:

$$
\left.\begin{array}{l}
f(t)=c_{i}^{*} \text { for } N \text { odd } \\
f(t)=0 \text { for } N \text { even }
\end{array}\right\},
$$

$N$ is the number of halfcycles and $t$ is given by the expression:

$$
\begin{aligned}
t= & (N-1) \tau+t^{\prime} \\
& 0<t^{\prime}<\tau .
\end{aligned}
$$

Then, for the first anodic halfcycle $(N=1)$, the classic solution for equation (2) for the concentration profile is obtained:

$$
c_{i}=c_{i}^{*}\left[1-\operatorname{erf} \frac{x}{2\left(D_{i} t^{\prime}\right)^{1 / 2}}\right] .
$$

As $c_{i}^{*}$ represents the equilibrium concentration of soluble species at the electrode surface, its value for a completely reversible reaction depends on $E_{a}$, the applied potential, as given by Nernst's equation for a single phase metal of unit activity:

$$
c_{i}^{*}=\exp \left[\frac{\mathrm{zF}}{\mathrm{R} T}\left(E_{\mathrm{z}}-E_{\mathrm{r}}^{0}\right)\right]
$$

where $E_{r}^{0}$ is the standard potential of the reaction, and the others symbols have their usual meaning.

Taking into account the boundary conditions (3) to (4) the following general analytical solutions comes out from the calculation procedure worked out in the appendix.

For the anodic halfcycle (or odd halfcycles):

$$
\begin{aligned}
& c_{i}\left(x,(N-1) \tau+t^{\prime}\right)= \\
& c_{i}^{*}\left\{1+\sum_{k=1}^{N}(-1)^{k} \operatorname{erf}\left[\frac{x}{2\left\{D_{i}\left|(k-1) \tau+t^{\prime}\right|\right\}^{1 / 2}}\right]\right\},
\end{aligned}
$$

and for the cathodic halfcycles (or even halfcycles)

$$
\begin{aligned}
& c_{i}\left(x,(N-1) \tau+t^{\prime}\right)= \\
& c_{i}^{*}\left\{\sum_{k=1}^{N}(-1)^{k+1} \operatorname{erf}\left[\frac{x}{2\left\{D_{i}\left|(k-1) \tau+t^{\prime}\right|\right\}^{1 / 2}}\right]\right\} .
\end{aligned}
$$

From the concentration profiles given by equations $(8)$ and (9) the corresponding fluxes of reacting species at the surface, expressed as anodic and cathodic current densities, $j_{\mathrm{a}}$ and $j_{\mathrm{c}}$, respectively, and charge densities for the anodic and cathodic halfcycles, $q_{\mathrm{a}}$ and $q_{\mathrm{c}}$, respectively, determined as a function of time and number of cycles can be immediately obtained. Thus:

$$
\begin{aligned}
j_{\mathrm{a}} & =-z_{\mathrm{i}} \mathrm{F} D_{\mathrm{i}}\left(\frac{\partial c_{i}}{\partial x}\right)_{x=0} \\
& =z_{i} \mathrm{~F} c_{i}^{*} D_{i}^{1 / 2} \pi^{-1 / 2} \sum_{k=1}^{N}(-1)^{k+1} \frac{1}{\left((k-1) \tau+t^{\prime}\right)^{1 / 2}}
\end{aligned}
$$

and:

$$
\begin{aligned}
j_{c} & =z_{i} F D_{i}\left(\frac{\partial c_{i}}{\partial x}\right)_{x=0} \\
& =z_{i} F c_{i}^{*} D_{i}^{1 / 2} \pi^{-1 / 2} \sum_{k=1}^{N}(-1)^{k} \frac{1}{\left((k-1) \tau+t^{\prime}\right)^{1 / 2}} .
\end{aligned}
$$

From equations (10) and (11) the values of $q_{\mathrm{a}}$ and $q_{\mathrm{c}}$ for an interval of time $\tau$, are calculated by integration as follows:

$$
q_{\mathrm{a}}=\left|\int_{0}^{z} j_{\mathrm{a}} \mathrm{d} t^{\prime}\right|
$$

and:

$$
q_{\mathrm{c}}=\left|\int_{0}^{\mathrm{r}} j_{\mathrm{c}} \mathrm{d} t^{\prime}\right| .
$$

Accordingly, the difference between $q_{\mathrm{a}}$ and $q_{\mathrm{c}}$ per cycle $\Delta q=q_{\mathrm{a}}-q_{\mathrm{c}}$ decreases progressively as the number of cycles increases, as given by the equation:

$$
\begin{aligned}
\Delta q= & z \mathrm{~F} \pi^{-1 / 2} D_{\mathrm{i}}^{1 / 2} c_{i}^{*} \tau^{1 / 2} \\
& \times \sum_{k=1}^{N}(-1)^{k+1}(\sqrt{k+1}-\sqrt{k}) .
\end{aligned}
$$

In this case, $\Delta q$, implies an accumulation of species $\mathbf{M}^{z+}$ in the solution per cycle. Likewise, the overall accumulation of charge, $\Delta q_{\mathrm{T}}$, after $N$ halfcycles results:

$$
\Delta q_{\mathrm{T}}=\sum_{k=1}^{N}\left(q_{\mathrm{a}}-q_{\mathrm{c}}\right)
$$

\section{BEHAVIOUR OF THE THEORETICAL MODEL}

The theoretical model predicts certain critical functionalities, some of them relevant from the standpoint of a direct comparison to experimental data.

Let us firstly consider on the basis of the theoretical equations the behaviour of the concentration of the soluble species as a function of three independent variables, namely, the distance perpendicular to the electrode, the time elapsed from the initiation of each pulse, and the frequency of square wave perturbing potential. 
The development of the concentration profile arising from the first anodic halfcycle, ie the initial halfcycle which produces through the reversible electrochemical reaction a source of diffusing species at $x=0$, is the classic one given by equation (6). The concentration profiles plotted as $c_{i}(x, t) / c_{i}^{*}$ us $x^{*}$, where $x^{*}=10 x / x_{\max }$ and $x_{\max }=4 \sqrt{D_{i} \tau}$, is shown in Fig. 1a. Otherwise, the development of the concentration profiles resulting from the cathodic halfcycle subsequent to the first anodic halfcycle is depicted in Fig. 2a. According to the boundary conditions imposed by the model, these profiles (cathodic halfcycle), initiated at $c_{i}(x, t) / c_{i}^{*}=0$, increase up to a maximum and reach again asymptotically $c_{i}(x, t) / c_{i}^{*}=0$ when $x^{*}$ increases. These profiles are broadened, the maximum $c_{i}(x, t) / c_{i}^{*}$ value decreases and appear at larger values of $x^{*}$ as $t^{\prime}$ increases. This behaviour results in a crossing of profiles. These effects are related to the fact that the decrease of $c_{i}(x, t) / c_{i}^{*}$ with $x^{*}$ becomes slower as $t^{\prime}$ increases.

The evolution of the concentration profile for each halfcycle during the subsequent cycles is considerably influenced by the preceding anodic and cathodic halfcycle. The greatest changes in the anodic profiles are produced in going from the first to the third cycle, and as the number of cycles fur ther increases the whole profile tends asymptotically to the stationary profile, but the values of $c_{i}(x, t) / c^{*}$ continues to increase with $N$ for large values of $x^{*}$. For a preset number of cycles, the inflexion points in Fig. 1b appear for greater

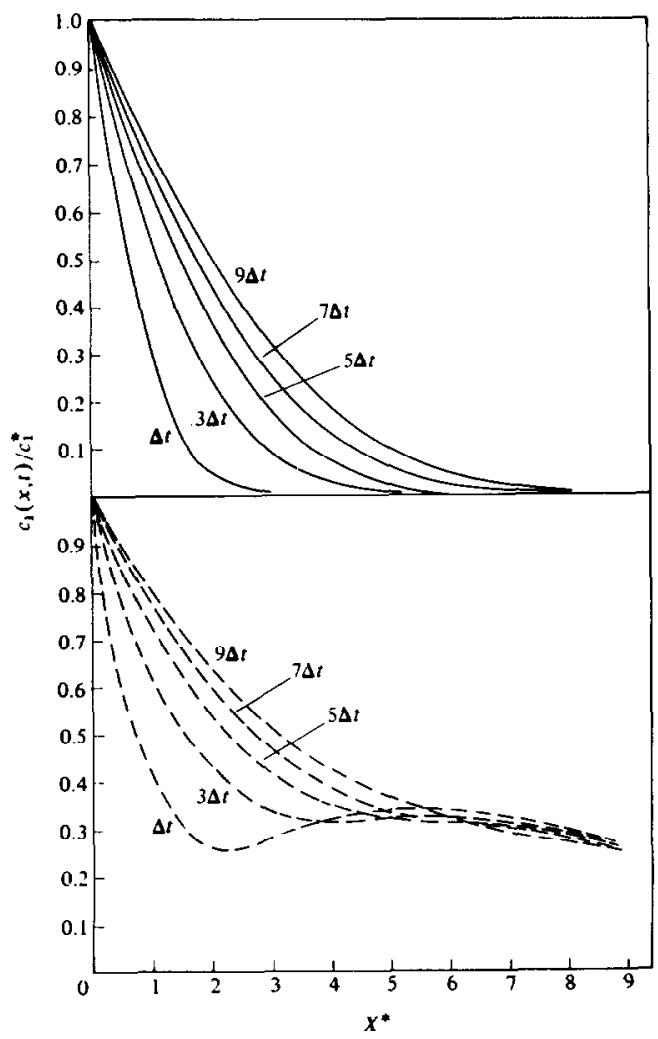

Fig. 1. Concentration profiles resulting from the model for the anodic halfcycles. $D_{i}=10^{-6} \mathrm{~cm}^{2} \mathrm{~s}^{-1} ; \Delta t=\tau / 10$. (a) $1^{\text {st }}$ halfcycle (anodic); (b) $19^{\text {th }}$ halfeycle (anodic)

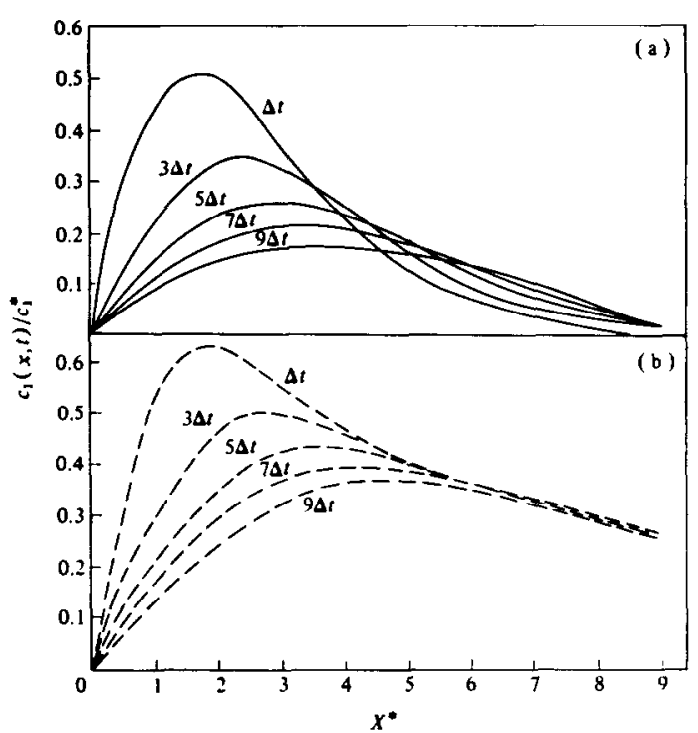

Fig. 2. Concentration profiles resulting from the model for the cathodic halfcycles. $D_{i}=10^{-6} \mathrm{~cm}^{2} \mathrm{~s}^{-1} ; \Delta t=\tau / 10$. (a) $2^{\text {nd }}$ halfcycle (cathodic); (b) $20^{\text {th }}$ halfcycle (cathodic).

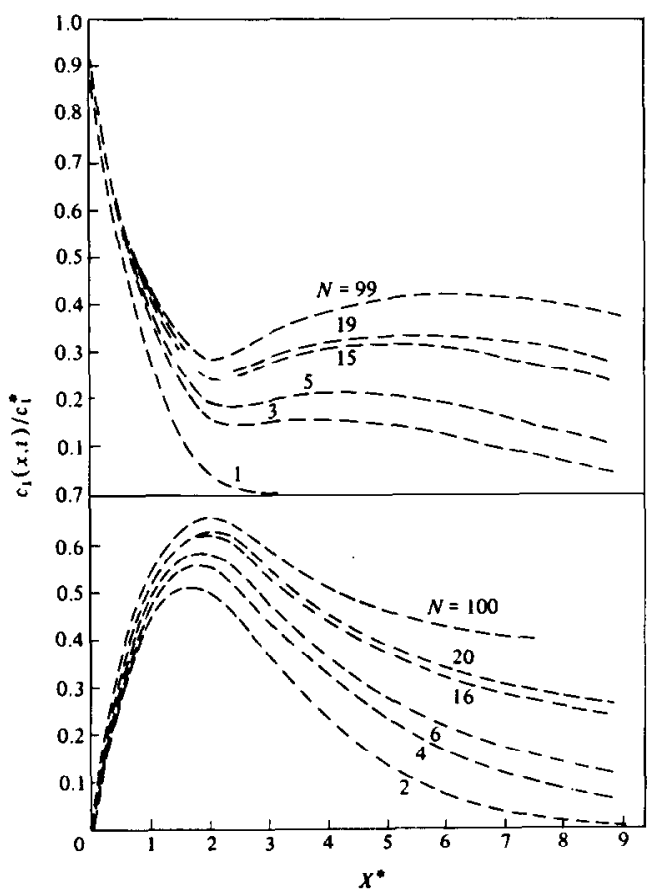

Fig. 3. Concentration profiles for $t=\Delta t$ for different cycles as identified by the numbers. (a) Anodic; (b) cathodic. $D_{i}=10^{-6} \mathrm{~cm}^{2} \mathrm{~s}^{-1} ; \Delta t=\tau / 10$.

values of $x^{*}$, as $t^{\prime}$ increases. Likewise, for a constant $t^{\prime}$ the inflection points remain at the same $x^{*}$ for any cycle as one should expect from the derivation of equations (8) and (9) with respect to $x^{*}$ (Fig. 3a).

Analogously, the concentration profiles resulting during the sequence of cathodic halfcycles show up a shift of the $c_{i}(x, t) / c_{i}^{*}$ maximum value towards greater values of $x^{*}$ with $t^{\prime}$ (Figs 2a and b). This shift with $t^{\prime}$ is 


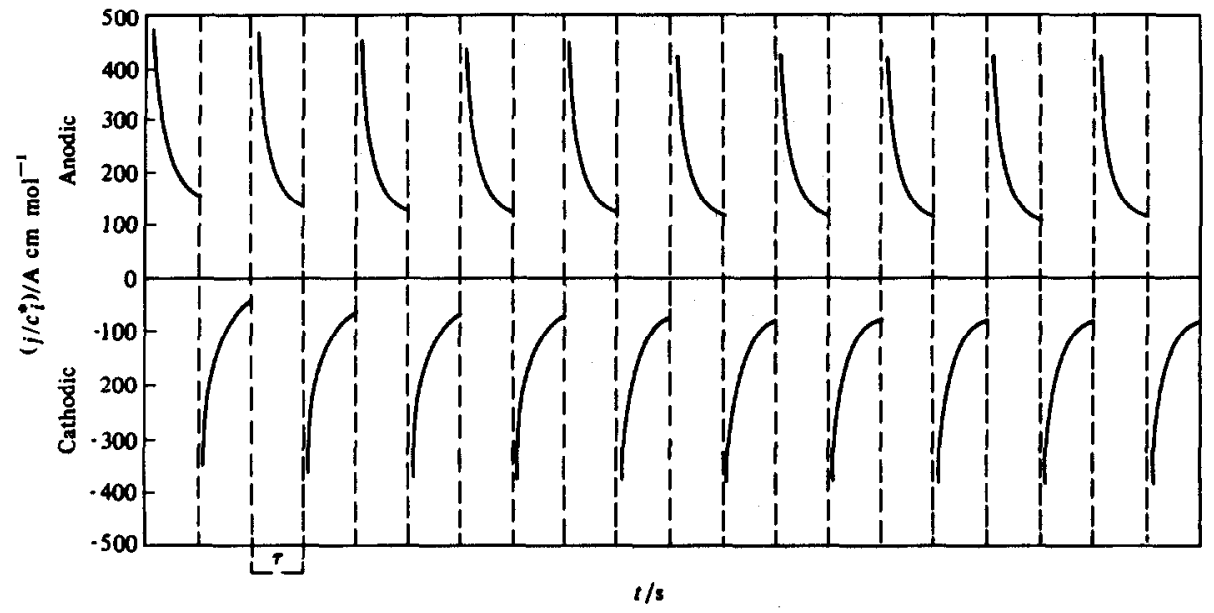

Fig. 4. Anodic and cathodic current transients for different halfcycles. $\tau=0.1 \mathrm{~s}$.

independent whether the first cathodic halfcycle or a large number of cathodic halfcycles are considered. Likewise, for a constant $t^{\prime}$, the maximal value of $c_{i}(x, t) / c_{i}^{*}$ changes slightly with $x^{*}$ but its decay becomes smoother as $N$ increases (Fig. $3 b$ ).

On the basis of the concentration profiles one can immediately derive the current transient behaviour associated with the reversible electrochemical reaction under the influence of the square wave perturbating potential. As shown in Fig. 4, the current transient either anodic or cathodic resulting during the corresponding halfcycle decay with time elapsed from the beginning of the halfcycle, but for a constant $t^{\prime}$ the cathodic current is smaller than the anodic one. This difference tends asymptotically to zero as the number of cycles, at a constant frequency, increases.

Similar relevant conclusions are accomplished for the charge resulting by integrating the current transients. Thus, the charge accumulated in each cycle decreases with $N$ in the anodic halfcycles and increases with $N$ in the cathodic halfcycles approaching asymptotically to a constant value as $N \rightarrow \infty$ (Fig. 5). Therefore, the charge difference, $\Delta q_{\mathrm{T}}$, as given by equation (15) between anodic and cathodic charges, results in the increase in concentration of the soluble product in the bulk of the solution. The value of $\Delta q_{\mathrm{T}}$ depends on the number of cycles considered and on the frequency (Fig. 6). This means that $\Delta q_{\mathrm{T}}$ for a given time $t$, increases as $f$ increases, although the opposite effect is observed when $\Delta q$ per cycle is considered.

According to equation (7) all the calculated magnitudes should depend principally on the value of the anodic potential step, ie the potential at which the soluble species is produced. Equation (8) holds, in principle, for any anodic potential provided that $j_{0} \rightarrow \infty$. Otherwise, the value of the cathodic potential step should approach $-\infty$ with respect to $E_{\mathrm{r}}$ to assure a null concentration of the reacting species at the electrode surface. When $j_{0} \rightarrow \infty$ this situation can be asymptotically reached for relatively small values of $E_{\mathrm{c}}$.

According to equation (8) when the potential step in the anodic half-cycle is considerably large, the value of $c_{i}^{*}$ can exceed the solubility product of species accumulated in the electrolyte solution. Nevertheless, the

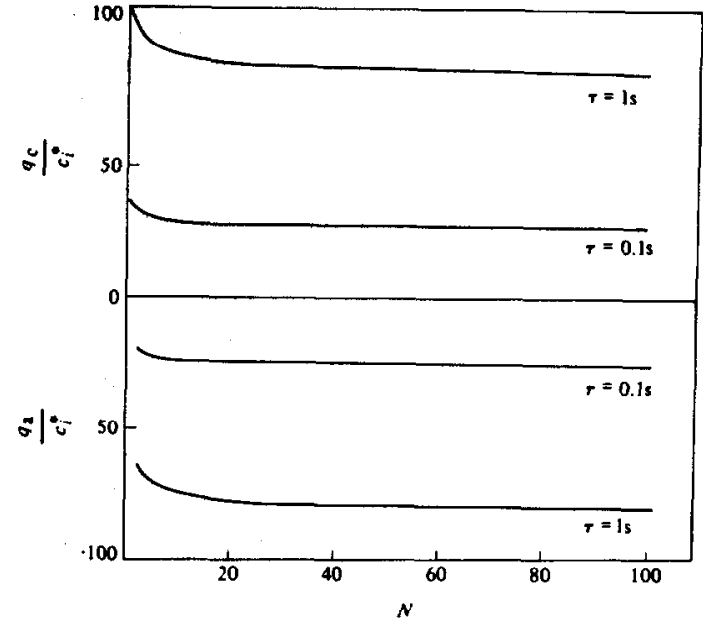

Fig. 5. Normalized charge densities $\left(q^{*}=q / c_{i}^{*}\right)$ for anodic and cathodic halfcycles $v s$ number of cycles: (๑) $\tau=0.1 \mathrm{~s}$; $(x) \tau=1 \mathrm{~s}$.

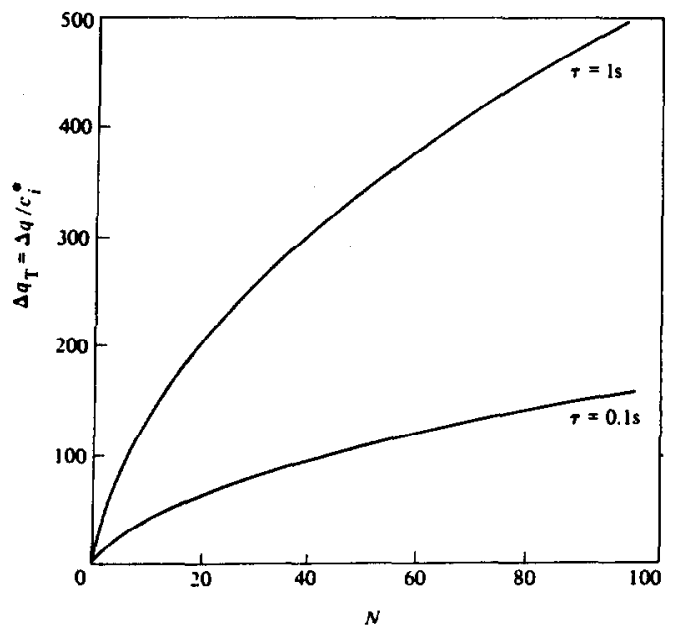

Fig. 6. $\Delta q_{\mathrm{T}}=\Delta q / c_{i}^{*} v s N$ plot for different frequencies. 
amount of accumulated product either as soluble species or insoluble species always is directly related to $\Delta q_{\mathrm{T}}$. For a preset potential cycling time, the narrower the period (the greater the frequency) of the symmetric perturbing potential, the greater the accumulation of product in the solution.

\section{CONCLUSION}

Most of the experimental data related to the electrochemical facetting of metals concern with the conditions for developing certain morphologies. There is only one paper dealing with the accumulation of soluble palladium species in solution during the electrochemical facetting treatments of this metal. These results refer to a repetitive square wave potential treatment made either for constant potential limits at different frequencies or at a constant frequency and variable potential windows[13]. These results, particularly those obtained at the lower frequencies, offer possibilities for making a preliminary comparison with the prediction of the model. It is clear that in agreement with the model there is an accumulation of soluble palladium during cycling, as it should correspond to equation (15).

The amount of soluble palladium accumulated in solution as the average amount of palladium dissolved per cycle decreases according to $t^{-1 / 2}$, whereas the amount of metal dissolved per unit time increases with $t^{\prime 1 / 2}$. These dependences are in qualitative agreement with equation (15) derived from the model.

A more exhaustive experimental work based on various redox system particularly useful for testing mass transfer models has already been published[15].*

Acknowledgement-This research project was financially supported by the Consejo Nacional de Investigaciones Cientificas y Técnicas and the Comissión de Investigaciones Cientificas de la Provincia de Buenos Aires.

This work was partially supported by the Regional Program for the Scientific and Technological Development of the Organization of the American States.

\section{REFERENCES}

1. A. J. Bard and L. R. Faukner, in Electrochemical Methods, Wiley, New York (1980).

2. E. Yeager and A. J. Salkin, in Techniques of Electrochemistry, Wiley, New York (1973).
3. A. R. Despic and K. I. Popov, J. appl. Electrochem. 1, 275 (1971).

4. N. Ibl, J. Cl. Puippe and H. Angerer, Surf. Technol. 6, 287 (1978).

5. K. Viswanathan and H. Y. Cheh, J. appl. Electrochem. 9, 537 (1979).

6. N. Ibl, Surf. Technol. 10, 81 (1980).

7. J. Cl. Puippe and N. Jbl, J. appl. Electrochem. 10, 775 (1980).

8. D-T. Chin, J. electrochem. Soc. 130, 1657 (1983).

9. C. Clerc and D. Landolt, J. appl. Electrochem. 17, 1144 (1987).

10. R. M. Cerviño, W. E. Triaca and A. J. Arvia, J. electrochem. Soc. 132, 266 (1984).

11. J. C. Canullo, W. E. Triaca and A. J. Arvia, J. electroanal. Chem. 175, 337 (1984).

12. A. Visintin, J. C. Canullo, W. E. Triaca and A. J. Arvia, J. electroanal. Chem. 239, 67 (1988)

13. C. L. Perdriel, E. Custidiano and A. J. Arvia, J. electroanal. Chem. 246, 165 (1988).

14. P. Delahay, New Instrumental Methods in Electrochemistry ( $3^{\text {rd }}$ edn), Interscience, New York (1962).

15. C. 1. Elsner, C. L. Perdriel, S. L. Marchiano and A. J. Arvia, Electrochim. Acta 35, 215 (1989).*

\section{APPENDIX}

The differential equation to be solved is:

$$
\mathrm{D} \frac{\partial^{2} c(x, t)}{\partial x^{2}}=\frac{\partial c(x, t)}{\partial t}
$$

with the boundary and initial conditions:

$$
\begin{aligned}
c(0, t) & =f(t) \\
c(x, 0) & =0, \\
\lim _{x \rightarrow \infty} c(x, t) & =0 .
\end{aligned}
$$

Applying the Laplace transform and taking into account the boundary conditions (2a) to (4a), one obtains:

$$
g(x, s)=L\{c(x, t)\}=L\{c(0, t)\} \exp (-x \sqrt{s / D}),
$$

and by carrying out the anti-transform, and by using the convolution theorem, the following general equation results:

$$
c(x, t)=\frac{x}{2 \sqrt{D N}} \int_{0}^{t} f(u) \frac{\exp \left[-x^{2} / 4 D(t-u)\right]}{(t-u)^{3 / 2}} \mathrm{~d} u,
$$

with $f(u)=f(t)$ as given by equations (3).

By integrating cycle by cycle [where $f(u)=c^{*}$ for odd cycles, and $f(u)=0$ for even cycles] from the first cycle to the $N$ cycle, and by considering $(k-1) \tau$ and $\tau$ as the integration limits, for each cycle $(k)$ equations $(8)$ and $(9)$ are obtained.

* Modelling of pulsating diffusional boundary layers--II by C. I. Elsner, C. L. Perdriel, S. L. Marchiano and A. J. Arvia appeared in Electrochimica Acta, Vol. 35, No. 1. 